\title{
Addressing Mental Health Needs of Health Care Workers through Peer Support Groups During the COVID-19 Crisis
}

Tanner Thornsberry', Jill Nault Connors ${ }^{2}$, Julie Welch ${ }^{3}$, Julie Hayden ${ }^{4}$, Jennifer Hartwell ${ }^{2}$, Michael Ober ${ }^{5}$, Sylk Sotto ${ }^{5}$, Claire Draucker ${ }^{6}$, Sally Wasmuth ${ }^{7}$, Malaz Boustani ${ }^{5}$, Ashley Overley $^{9}$, Patrick Monahan ${ }^{10}$, Kurt Kroenke ${ }^{5}$

${ }^{1}$ Indiana University School of Medicine, ${ }^{2}$ IUSM Department of Surgery, ${ }^{3}$ IUSM Department of Emergency Medicine, ${ }^{4}$ National Alliance on Mental IIIness Greater Indianapolis, ${ }^{5}$ IUSM Department of Medicine, ${ }^{6}$ Indiana University School of Nursing, ${ }^{7}$ IUPUI School of Health \& Human Sciences, ${ }^{9}$ IUSM Department of Psychiatry, ${ }^{10}$ IUSM Department of Biostatistics

\section{Background:}

The COVID-19 pandemic has placed a high psychological burden on frontline healthcare workers. Often quarantined away from their families and with little downtime to process their experiences, those on the frontlines of the pandemic are experiencing pronounced levels of distress and significantly elevated rates of burnout. Although many wellness and psychological resources are available to providers, there is low uptake and little is known about their effectiveness. In this study, we assess the impact of group-based peer support sessions on symptoms of acute distress, anxiety and depression, and provider burn out.

\section{Methods:}

An established peer support model is adapted for use with groups of frontline healthcare workers that will participate in 6 to 8 weekly group sessions using videoconferences. The study approach is a phased feasibility to research design. During the feasibility phase, we will initially test the intervention in 3 groups of 8 providers using a quasi-experimental, pre- post analysis of change. If preliminary results are positive, we will scale the intervention and progress to a more rigorous study design using a differences-in-differences approach to assess change over time between exposure and non-exposure groups. Outcomes will be measured at baseline, intervention completion, and 3- and 6- month follow ups. During the feasibility phase we will assess intervention fidelity and conduct qualitative analysis to assess the effects of the pandemic on work, family and social life.

\section{Results:}

This is a work in progress. At present we have recruited 28 emergency medicine physicians and residents with a target start date of August 1, 2020.

\section{Potential Impact:}

We anticipate the results of this study will provide evidence in support of a recent call for "the use of non-clinical mental health support, such as social or peer support" from the American College of Emergency Physicians in conjunction with 42 leading professional organizations in medicine. In addition, results may lead to advocacy for improved policies that mitigate against "fear of resultant loss of licensure, loss of income, or other career setbacks" for seeking mental health support. 